\title{
VARIABILIDADE ESPACIAL DA UMIDADE DE UM LATOSSOLO VERMELHO- AMARELO SOB PLANTIO DIRETO ${ }^{1}$
}

\author{
FABRÍCIO DE M. T. SAMPAIO ${ }^{2}$, MOZART M. FERREIRA ${ }^{3}$, \\ MARCELO S. DE OLIVEIRA ${ }^{4}$, ALBERTO C. ALMEIDA ${ }^{5}$, MARCOS R. JOSÉ ${ }^{6}$
}

\begin{abstract}
RESUMO: O estudo da dependência espacial de atributos do solo é importante para aprimorar as práticas de manejo e de amostragem. Este trabalho teve o objetivo de estudar a variabilidade espacial da umidade de um Latossolo Vermelho-Amarelo sob plantio direto, cultivado com milho, e sua relação com o carbono orgânico total do solo, macroporosidade e microporosidade. A pesquisa foi conduzida em uma área localizada no município de Lavras - MG. Na avaliação dos atributos do solo, foi delineada uma malha experimental com dimensões iguais a 90 x $90 \mathrm{~m}(0,81$ ha de área), com espaçamento entre os pontos de amostragem de 15 x $15 \mathrm{~m}$. Para obter maior detalhamento da dependência espacial dos dados, realizou-se a implantação de mais duas malhas (zoom) dentro de um quadrante da grande malha, totalizando 73 pontos de leitura. A umidade do solo em base volume $\left(\mathrm{cm}^{3} \mathrm{~cm}^{-3}\right)$ foi determinada in situ em duas datas, utilizando-se de um equipamento de TDR. Por meio da análise dos dados das duas datas pela Estatística Clássica, observaram-se coeficientes de variação médios para a umidade do solo. Obteve-se um semivariograma com efeito pepita puro quando o solo se apresentou mais úmido, indicando a ausência total de estruturação nestas condições. Já para o solo mais seco, ajustou-se o modelo exponencial ao semivariograma, o qual mostrou moderada dependência espacial e alcance de $75 \mathrm{~m}$. Verificou-se que a associação entre a umidade do solo e o carbono orgânico aumentou na condição de solo mais seco.
\end{abstract}

PALAVRAS-CHAVE: geoestatística, manejo do solo, reflectometria no domínio do tempo.

\section{SPATIAL VARIABILITY OF SOIL WATER CONTENT OF A RED-YELLOW LATOSOL UNDER NO-TILLAGE}

\begin{abstract}
The study of spatial dependence of soil attributes is important to improve management practices and sampling. This research aimed to investigate the spatial and temporal variability of the soil water content of a Red-Yellow Latosol under no-tillage cultivated with maize and its relation to the total organic carbon in soil, macroporosity and microporosity. The experiment was on experimental area located in Lavras, state of Minas Gerais, Brazil, which was implanted an experimental mesh with dimensions $90 \times 90 \mathrm{~m}$ (0.81 ha of area) and spacing among points equal to $15 \times 15 \mathrm{~m}$. For a more detailed spatial dependence of data, it was realized the introduction of two more meshes (zoom) in a quadrant of the great mesh, totaling 73 reading points. The soil water content in base volume $\left(\mathrm{cm}^{3} \mathrm{~cm}^{-3}\right)$ was determined on two dates, using an equipment that is based on the technique of Time Domain Reflectometry (TDR). Through the analysis of data from two dates with classical statistical, it was observed average coefficients of variation for soil moisture. It was obtained a semivariogram with pure nugget effect to a wetter soil, indicating a total lack of structure for soil moisture. For the drier soil, it was found that the association between soil moisture and organic carbon increased and fit the exponential model to semivariogram, which showed moderate spatial dependence and range equal to $75 \mathrm{~m}$.
\end{abstract}

KEYWORDS: geostatistic, soil management, time domain reflectometry.

\footnotetext{
${ }^{1}$ Extraído da Tese de Doutorado em Ciência do Solo do primeiro autor.

${ }^{2}$ Eng $^{\mathrm{o}}$ Agrícola, Prof. Adjunto I, CPCE - Universidade Federal do Paiuí, Bom Jesus - PI, Fone: (0XX89) 3562.2535, flabricio@gmail.com.

${ }^{3}$ Eng $^{\mathrm{O}}$ Agrônomo, Prof. Titular, Universidade Federal de Lavras, DCS, Lavras - MG.

${ }^{4}$ Eng ${ }^{0}$ Agrícola, Prof. Associado, Universidade Federal de Lavras, DEX, Lavras - MG.

${ }^{5}$ Graduando em Eng ${ }^{a}$ Agrícola, Universidade Federal de Lavras, DCS, Lavras - MG.

${ }^{6}$ Eng $^{\mathrm{o}}$ Agrônomo, Pesquisador, Universidade Federal de Lavras, DCS, Lavras - MG.

Recebido pelo Conselho Editorial em: 25-8-2010
}

Aprovado pelo Conselho Editorial em: 20-7-2010 


\section{INTRODUÇÃO}

O sistema plantio direto (SPD) é uma das práticas de manejo do solo que tem a função de conservar o solo, visando à sustentabilidade da produção agrícola. Este sistema de manejo tem como pilares o mínimo revolvimento do solo, a cobertura vegetal continuada do solo e a rotação de culturas (STONE et al., 2006; WEIRICH NETO et al., 2006; REIS et al., 2007).

O conhecimento da distribuição espacial dos atributos do solo em determinada área é importante para o refinamento das práticas de manejo e avaliação dos efeitos da agricultura sobre a qualidade ambiental (CAMBARDELLA et al., 1994). Também tem importância na definição da intensidade de amostragem do solo para sua caracterização, possibilitando, dessa maneira, reduzir o erro-padrão da média, maximizando a eficiência da amostragem, e reduzir os custos e a mão de obra (WEBSTER \& OLIVER, 2001).

A umidade volumétrica do solo $(\theta)$ no campo pode ser avaliada por métodos diretos e indiretos. Dentre os métodos diretos, o gravimétrico é considerado altamente confiável e, consequentemente, preferido (TEIXEIRA et al., 2003). Uma das desvantagens deste método são a grande demanda de trabalho para a amostragem do solo e os procedimentos posteriores no laboratório, uma vez que, por ser um método destrutivo, não permite retirar nova amostra no mesmo local, posteriormente. Ultimamente, a técnica da Reflectometria no Domínio do Tempo (TDR) vem sendo amplamente usada como método indireto não destrutivo para a avaliação da umidade do solo.

A umidade do solo apresenta elevada variação no espaço como consequência da variabilidade de outras propriedades. O estudo detalhado do seu comportamento é necessário para se saber como ela varia no campo, além de fornecer elementos essenciais ao estabelecimento ou aprimoramento de práticas de manejo agrícola que visem à otimização da produtividade (GREGO et al., 2006).

A Geoestatística, ferramenta estatística utilizada para estudar a variabilidade espacial, possibilita a interpretação dos resultados com base na estrutura da variabilidade natural dos atributos avaliados, considerando a dependência espacial dentro do intervalo de amostragem (ANDRIOTTI, 2003).

Os estudos da variabilidade espacial, utilizando a geoestatística, têm sido realizados através da análise de semivariogramas (ENGHBALL et al., 1999). A obtenção de semivariogramas representativos depende fundamentalmente do número de pares de pontos encontrado em determinada direção, para diferentes distâncias (WEBSTER \& OLIVER, 2001).

GREGO et al. (2006), estudando um Latossolo Vermelho eutrófico sob plantio direto, citam que a dependência espacial é mais fraca quando se têm valores mais baixos de umidade do solo. Já VERONESE JÚNIOR et al. (2006) observaram que, próximo da capacidade de campo, os semivariogramas mostram efeito pepita puro para a umidade do solo, a qual depende da distribuição espacial dos macroporos e microporos.

Neste trabalho, teve-se o objetivo de aplicar técnicas da estatística clássica e da geoestatística para estudar a variabilidade espacial da umidade do solo de Latossolo Vermelho-Amarelo cultivado com milho sob plantio direto, em duas datas de amostragem, e sua relação com o carbono total do solo.

\section{MATERIAL E MÉTODOS}

A pesquisa foi realizada no sítio Olho d'Água, localizado no município de Lavras (MG), em uma área de cultivo de milho manejado com o sistema de plantio direto, desde outubro de 1998, envolvendo cobertura de inverno, tendo o milho como cultura principal. A propriedade possui 27 ha de área total, localizando-se a $21^{\circ} 16^{\prime} 15^{\prime \prime}$ de latitude sul e 44⒌'22' de longitude oeste.

O tipo climático da região é $\mathrm{Cwb}$, segundo a classificação de Köeppen, com chuvas concentradas entre novembro e março. $\mathrm{O}$ solo onde a malha experimental foi instalada, classificado 
como Latossolo Vermelho-Amarelo (LVA) com textura argilosa, encontra-se na área mais plana da propriedade, a $950 \mathrm{~m}$ de altitude.

O manejo de plantas daninhas foi realizado com o auxílio dos herbicidas glifosate, aplicado em pós-colheita e anteriormente ao plantio do milho; e Atrazina, aplicada em pós-emergência, entre 15 e 20 dias após o plantio. As culturas escolhidas para a cobertura de inverno, após três anos de testes com várias espécies, foram o nabo forrageiro e o tremoço-branco, pois estas apresentaram melhor associação entre si e maior adaptabilidade às condições edafoclimáticas da propriedade.

A grande malha experimental apresenta dimensões iguais a 90 x $90 \mathrm{~m}(0,81$ ha de área). O espaçamento entre os pontos de leitura adotados foi de 15 x $15 \mathrm{~m}$, com um subtotal de 49 pontos. Para obter maior detalhamento da dependência espacial dos dados, realizou-se a implantação de mais duas malhas (zoom) dentro de um quadrante da grande malha escolhido por meio de sorteio, excetuando-se aqueles das bordas (Figura 1). Os espaçamentos utilizados para as malhas do zoom foram de 5 x $5 \mathrm{~m}$ e 1,67 x 1,67 m, totalizando mais 24 pontos de leitura no total.

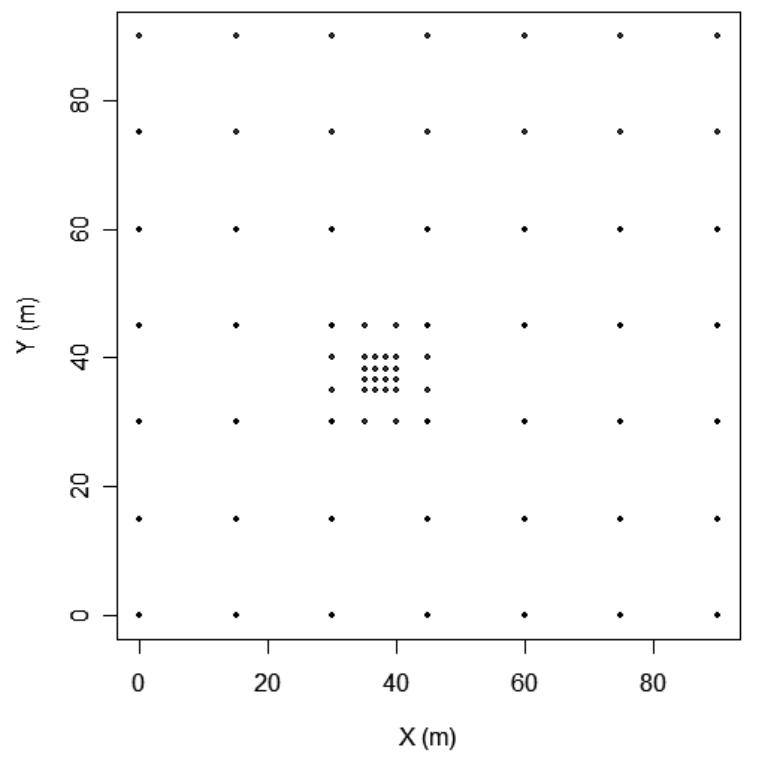

FIGURA 1. Grid de amostragem utilizado para a obtenção da umidade do solo. Sampling grid used to obtain soil moisture.

Para a estimativa da umidade do solo $\left(\mathrm{cm}^{3} \mathrm{~cm}^{-3}\right)$, utilizou-se a constante dielétrica (ka) obtida por um aparelho que utiliza a técnica da Reflectometria no Domínio do Tempo (TDR). A sonda de leitura apresenta $15 \mathrm{~cm}$ de comprimento com duas hastes não segmentadas. O TDR foi calibrado para a obtenção dos valores de umidade no solo estudado, utilizando metodologia similar à de SOUZA et al. (2006), sendo as medidas realizadas nos dias 13 de abril e 19 de maio de 2007.

Em cada ponto de amostragem, foram retiradas amostras de solo deformadas e indeformadas em duas profundidades $(0-7,5 \mathrm{~cm}$ e 7,5-15 cm). Estas destinaram-se à determinação dos teores de carbono orgânico do solo, conforme metodologia de TEDESCO et al. (1995), além da macroporosidade e microporosidade, atributos que apresentam elevada influência no armazenamento de água no solo.

Os dados foram analisados no programa SISVAR v 4.3, usando a Estatística Clássica para descrever adequadamente a tendência central e avaliar a hipótese de normalidade dos dados (FERREIRA, 2000). Foi realizada também a análise de correlação entre os atributos estudados com auxílio do software SAEG versão 9.0. A dispersão dos dados e a variabilidade espacial foram analisadas usando a Geoestatística, através de parâmetros obtidos pelo programa GeoR v. 2.4.1 (RIBEIRO JR. \& DIGGLE, 2001) do software R. Os modelos de semivariograma que apresentaram menor soma de quadrados pelo método dos mínimos quadrados ordinários, foram os utilizados. 


\section{RESULTADOS E DISCUSSÃO}

Na Tabela 1, é apresentado o resultado do resumo estatístico da análise do comportamento da umidade do solo. Observa-se que os valores de umidade do solo apresentaram assimetria e curtose compatíveis com a distribuição normal somente para a primeira data de amostragem. Valores próximos de zero para assimetria e de três para a curtose, a qual mede o grau de achatamento, caracterizam a distribuição normal de frequências (FERREIRA, 2000).

TABELA 1. Parâmetros estatísticos para a umidade do solo $\left(\mathrm{cm}^{3} \mathrm{~cm}^{-3}\right)$. Statistical parameters for soil moisture $\left(\mathrm{cm}^{3} \mathrm{~cm}^{-3}\right)$.

\begin{tabular}{|c|c|c|c|c|c|c|c|}
\hline Data & $\begin{array}{c}\text { Média } \\
\left(\mathrm{cm}^{3} \mathrm{~cm}^{-3}\right)\end{array}$ & Desvio-Padrão & $\begin{array}{l}\text { C.V. } \\
(\%)\end{array}$ & $\begin{array}{r}\text { Máximo } \\
---\mathrm{cm}\end{array}$ & $\begin{array}{l}\text { Mínimo } \\
-3---\end{array}$ & Assimetria & Curtose \\
\hline 13-04-2007 & 0,31 & 3,14 & 10,1 & 0,39 & 0,24 & 0,01 & 2,88 \\
\hline 19-05-2007 & 0,19 & 2,44 & 12,8 & 0,26 & 0,145 & 0,85 & 3,21 \\
\hline
\end{tabular}

C.V. - coeficiente de variação.

Em relação aos coeficientes de variação, nas duas datas, foram verificados valores dentro do intervalo de 10 a 20\%, sendo estes valores considerados médios, segundo PIMENTEL-GOMES (1984). Deve-se salientar que a Estatística Clássica avalia os dados considerando a variável independente espacialmente, por isso ela deve ser usada com cautela se os dados apresentarem estrutura de dependência espacial. Se esta dependência ocorrer, verificada pela Geoestatística, a interpretação dos dados pela Estatística Clássica fica tendenciosa.

Nas Figuras 2a e 2b, apresentam-se os semivariogramas obtidos para as umidades volumétricas nas duas datas. Observa-se o efeito pepita puro para a primeira amostragem (Figura 2a), que resulta em atribuir os mesmos pesos a todos os pontos de amostragem, ou seja, pontos mais próximos não têm maior influência no valor estimado, o que se resume em desconsiderar a correlação espacial (ANDRIOTTI, 2003). Já para a condição de solo mais seco, ajustou-se o modelo exponencial ao semivariograma obtido através dos dados, indicando que a variabilidade espacial da umidade é dependente da magnitude dos seus valores em função das condições pluviométricas da época de amostragem (Figura 2b).
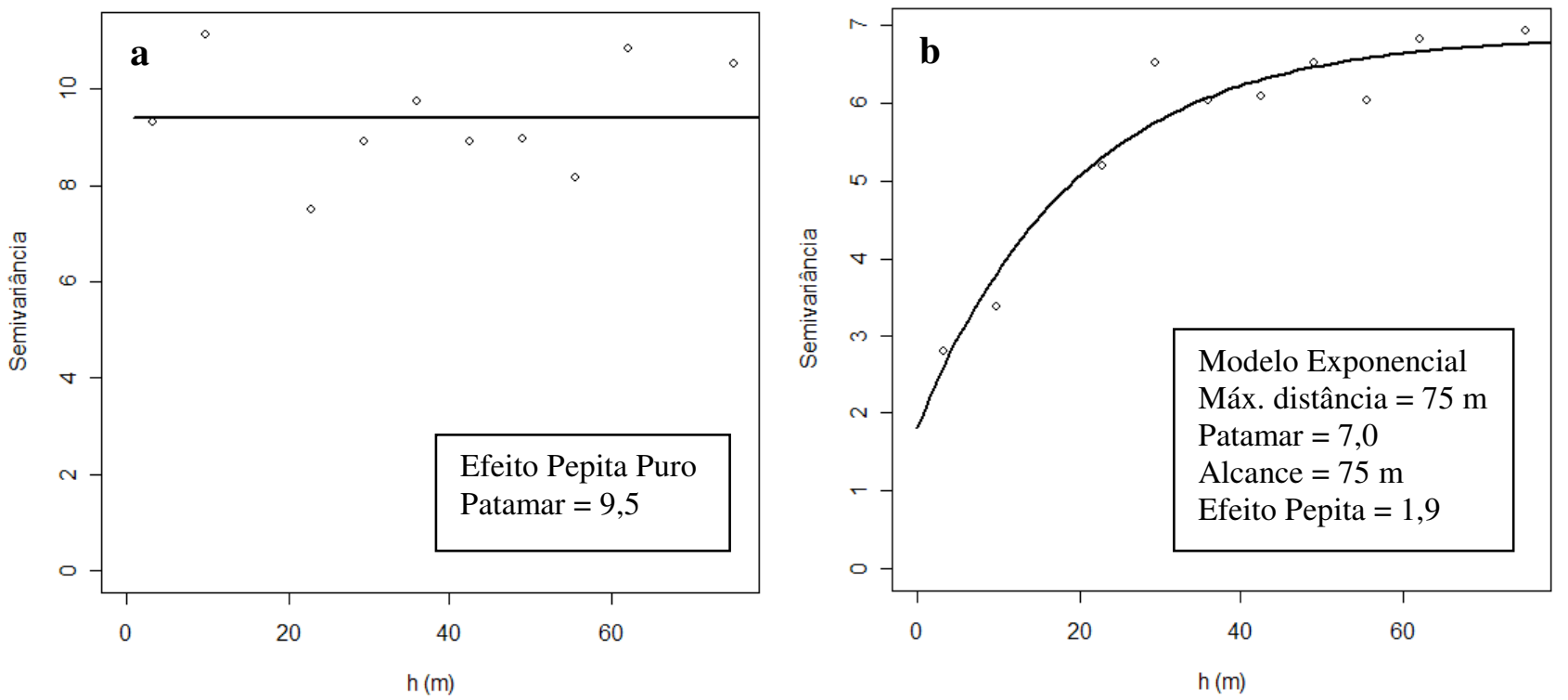

FIGURA 2. Semivariogramas para os dados de umidade do solo ajustados por meio dos mínimos quadrados ordinários (a-13-4-2007; b-19-5-2007). Semivariograms for data on soil moisture adjusted by ordinary least squares (a- 13/04/2007; b- 19/05/2007).

O efeito pepita puro indica a ausência total de estruturação do fenômeno estudado (ANDRIOTTI, 2003). Nessa situação, os resultados obtidos pela Geoestatística será os mesmos 
indicados pela Estatística Clássica, a qual assume que a variação das características do solo dentro das unidades amostrais não são correlacionadas, e que o valor médio se constitui no melhor estimador das mesmas em qualquer local da unidade amostral.

GREGO et al. (2006) não detectaram dependência espacial para a umidade do solo de amostras coletadas a 30 e $90 \mathrm{~cm}$ de profundidade, em um Latossolo Vermelho eutroférrico sob plantio direto, ressaltando que a densidade de amostragem pode ter sido insuficiente para expressar a variabilidade espacial destas amostras. No entanto, SOUZA et al. (2005), trabalhando em um Latossolo Vermelho eutroférrico sob cultivo de cana-de-açúcar, encontraram forte dependência espacial para a umidade do solo na profundidade de 0 a 0,2 m. Resultados semelhantes aos de SOUZA et al. (2005) foram encontrados por GREGO \& VIEIRA (2005) em estudos realizados em um Latossolo Vermelho sob preparo convencional. As diferenças em relação ao presente trabalho, no comportamento das observações da umidade do solo, podem estar relacionadas às diferenças existentes no ambiente edáfico dos sistemas de manejo adotados nas áreas estudadas.

A ausência de estrutura de dependência espacial encontrada neste trabalho para a primeira data também pode estar associada aos elevados valores de umidade obtidos na área estudada (média igual a $0,31 \mathrm{~cm}^{3} \mathrm{~cm}^{-3}$ ), pois as leituras foram executadas três dias após a ocorrência de uma precipitação pluviométrica acumulada de $70 \mathrm{~mm}$, medida por meio de um pluviômetro instalado na propriedade. Dessa maneira, encontra-se maior homogeneidade nos valores de umidade do solo, evidenciada pelo menor C.V. (Tabela 1), pois os atributos do solo, tais como a microporosidade e teor de matéria orgânica, ainda não se expressaram sobre a retenção de água do solo. Porém, por não existir estrutura, a previsibilidade do fenômeno fica dificultada.

Segundo a classificação de CAMBARDELLA et al. (1994), o grau de dependência espacial verificado para a condição de solo mais seco, obtido através da relação entre o efeito pepita e o patamar, demonstrou moderada dependência espacial para o semivariograma estudado (valor igual a $73 \%$ ), com alcance igual a $75 \mathrm{~m}$. GREGO et al. (2006) também obtiveram semivariogramas com moderada dependência espacial para todas as épocas estudadas nas amostragens a $60 \mathrm{~cm}$ de profundidade em área sob plantio direto, com alcance de 90 a $110 \mathrm{~m}$. O estudo dos semivariogramas para o teor de carbono orgânico do solo, macro e microporosidade está apresentado na Tabela 2. Observa-se que a microporosidade, na camada de $0-7,5 \mathrm{~cm}$, foi o atributo que demonstrou parâmetros geoestatísticos mais próximos dos encontrados para a umidade do solo mais seco, com alcance igual a $84 \mathrm{~m}$ e moderada dependência espacial. $\mathrm{O}$ teor de carbono orgânico do solo, na camada de 7,5-15 cm também apresentou moderada dependência espacial dos dados.

TABELA 2. Valores do efeito pepita $\left(\mathrm{C}_{0}\right)$, patamar $\left(\mathrm{C}_{0}+\mathrm{C}_{1}\right)$, alcance (a) e classificação dos modelos ajustados para os teores de carbono orgânico do solo (COT), macroporosidade e microporosidade. Values of nugget effect $(\mathrm{CO})$, sill $(\mathrm{CO}+\mathrm{C1})$, range (a) and classification of models adjusted for organic carbon content of soil (TOC), macroporosity and microporosity.

\begin{tabular}{cclcccc}
\hline $\begin{array}{c}\text { Profundidade } \\
(\mathrm{cm})\end{array}$ & Atributo & Modelo & $\mathrm{C}_{0}$ & $\mathrm{C}_{0}+\mathrm{C}_{1}$ & $\mathrm{a}(\mathrm{m})$ & $\begin{array}{c}\text { Dependência } \\
\text { Espacial }\end{array}$ \\
\hline \multirow{3}{*}{$0-7,5$} & COT & Exponencial & 0,042 & 0,059 & 12,8 & Moderada \\
& Macro & Exponencial & 15,530 & 20,340 & 39,6 & Fraca \\
& Micro & Exponencial & 3,32 & 8,93 & 84 & Moderada \\
\hline \multirow{3}{*}{$7,5-15$} & COT & Exponencial & 0,024 & 0,073 & 38,7 & Moderada \\
& Macro & EPP & - & 17,24 & - & - \\
& Micro & EPP & - & 3,45 & - & - \\
\hline
\end{tabular}

EPP - efeito pepita puro

Os dados demonstram, em sua maior parte, distribuição espacial aleatória, indicando elevada variabilidade do solo para esses atributos. Verificou-se ausência de dependência espacial para a macroporosidade e microporosidade do solo na camada de 7,5-15 $\mathrm{cm}$. As causas adicionais de 
variabilidade das propriedades físicas nos cultivos conservacionistas, como a falta de revolvimento do solo e tráfego aleatório do maquinário agrícola, podem ser apontadas como fatores condicionantes para o comportamento encontrado nesta pesquisa.

Por meio da interpolação dos pontos por meio da krigagem, obtiveram-se os mapas temáticos com legenda para a umidade do solo na segunda data de amostragem, macro e microporosidade na camada de 0-7,5 cm, e teor de carbono orgânico do solo nas duas camadas (Figura 3). Estes mapas facilitam a visualização da distribuição espacial das variáveis e auxiliam no planejamento do manejo da área.
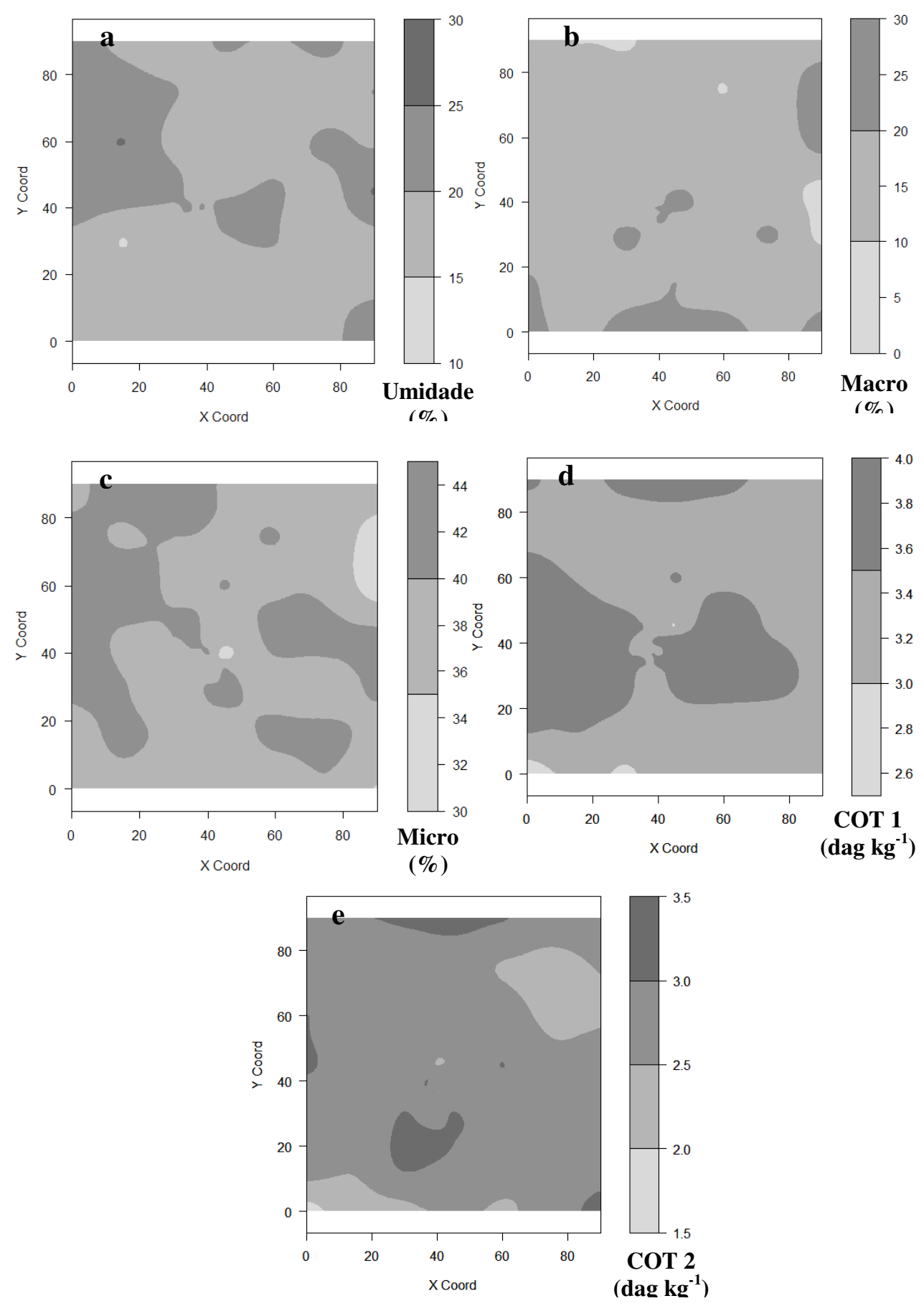

FIGURA 3. Mapas de krigagem para a umidade do solo (a), Macroporosidade 0-7,5 cm (b), Microporosidade 0-7,5 cm (c) e teor de carbono orgânico do solo nas camadas de 07,5 (d) e 7,5-15 cm (e). Kriging maps for soil moisture (a), macroporosity $\mathbf{0 - 7 , 5} \mathbf{~ c m}$ (b), Microporosity $0-7.5 \mathrm{~cm}$ (c) and organic carbon content in the soil layers 0 $7.5(\mathrm{~d})$ and $7,5-15 \mathrm{~cm}(\mathrm{e})$. 
Verifica-se a predominância de valores mais elevados de umidade do solo na região superior esquerda da área estudada, coincidindo com pontos de maior microporosidade. Os elevados valores nas outras regiões podem estar associados também a outros atributos, como o teor carbono orgânico total.

Na Tabela 3, verificam-se os coeficientes de correlação entre os valores de umidade do solo e os teores de carbono orgânico, macroporosidade e microporosidade, nas camadas de 0-7,5 cm e 7,5$15 \mathrm{~cm}$.

TABELA 3. Coeficientes de correlação para a umidade do solo $\left(\mathrm{cm}^{3} \mathrm{~cm}^{-3}\right)$ com outros atributos físicos do solo nas duas profundidades amostradas. Correlation coefficients for soil moisture $\left(\mathrm{cm}^{3} \mathrm{~cm}^{-3}\right)$ with other soil physical attributes in the two depths sampled.

\begin{tabular}{ccccccc}
\hline \multirow{2}{*}{ Data } & \multicolumn{5}{c}{$0-7,5 \mathrm{~cm}$} & $7,5-15 \mathrm{~cm}$ \\
\cline { 2 - 7 } & COT & Macro & Micro & COT & Macro & Micro \\
\hline $13-4-2007$ & $-0,04$ & 0,21 & $-0,12$ & $-0,01$ & 0,09 & $-0,02$ \\
\hline $19-5-2007$ & 0,17 & $-0,07$ & 0,01 & 0,25 & $-0,15$ & 0,09 \\
\hline Ctotal - teor de carbono orgânico total; Macro - macroporosidade; Micro - microporosidade.
\end{tabular}

$\mathrm{Na}$ condição de solo mais úmido (13-04-2007), o atributo que mais se associou com a retenção de água foi a macroporosidade, enquanto para o solo mais seco, os teores de carbono orgânico foram mais relevantes. Quando a umidade do solo se apresenta abaixo da capacidade de campo, a capilaridade é a força que predomina na retenção de água no solo (FERREIRA \& DIAS JUNIOR, 1997). Nota-se tendência de aumento da correlação entre a umidade do solo e os teores de carbono orgânico do solo e microporosidade à medida que o solo se torna mais seco. Esta maior associação se justifica, pois a matéria orgânica e os microporos apresentam elevada capacidade de reter água. Portanto, quando o solo passa para um estado mais seco, as regiões com maior concentração de matéria orgânica e de microporos tendem a apresentar maiores valores de umidade.

\section{CONCLUSÕES}

A presença da estrutura de dependência espacial da umidade do solo está associada às condições de umidade do solo, não sendo detectada dependência no período mais úmido.

Na condição de solo mais seco, observou-se moderada dependência espacial para a umidade do solo com alcance igual a $75 \mathrm{~m}$.

A associação entre a umidade do solo e o carbono orgânico aumentou na condição de solo mais seco.

\section{AGRADECIMENTOS}

À Fundação de Amparo à Pesquisa do Estado de Minas Gerais (FAPEMIG), pela concessão da bolsa do primeiro autor e pelo auxílio financeiro para o trabalho, e ao Eng ${ }^{\circ}$ Agrônomo Marcos Roveri José, por ceder e manter a área para a pesquisa.

\section{REFERÊNCIAS}

ANDRIOTTI, J. L.S. Fundamentos de estatística e geoestatística. São Leopoldo: Ed. Unisinos, 2003. $165 \mathrm{p}$.

CAMBARDELLA, C. A.; MOORMAN, T. B.; NOVAK, J. M.; PARKIN, T. B.; KARLEN, D. L.; TURCO, R. F.; KONOPKA, A. E. Field-scale variability of soil properties in central Iowa soils. Soil Science Society of America Journal, Madison, v.58, p.1.501-1.511, 1994.

ENGHBALL, B.; HERGERT, G.W.; LESOING, G.W.; FERGUSON, R.B. Fractal analysis of spatial and temporal variability. Geoderma, v.88, p.349-362, 1999. 
FERREIRA, D.F. Análises estatísticas por meio do SISVAR para Windows 4.0. In: REUNIÃO ANUAL DA REGIÃO BRASILEIRA DA SOCIEDADE INTERNACIONAL DE BIOMETRIA, 45., 2000, São Carlos. Anais... São Carlos: Universidade Federal de São Carlos, 2000. p.255-258.

FERREIRA, M.M.; DIAS JÚNIOR, M.S. Física do solo. Lavras: UFLA/FAEPE, 1997. 63 p. GEOR, The geoR package. Disponível em: www.est.ufpr.br/geoR. Acesso em 29 ago 2007.

GREGO, C.R.; VIEIRA, S.R. Variabilidade espacial de propriedades físicas do solo em uma parcela experimental. Revista Brasileira de Ciência do Solo, Viçosa - MG, v.29, n.1, p.169-177, 2005.

GREGO, C.R.; VIEIRA, S.R.; ANTONIO, A.M.; DELLA ROSA, S.C. Geostatistical analysis for soil moisture content under the no tillage cropping system. Scientia Agricola, Piracicaba, v.63, n.4, p.341-350, 2006.

PIMENTEL-GOMES, F. A estatística moderna na pesquisa agropecuária. Piracicaba: Associação Brasileira para Pesquisa da Potassa e do Fosfato, 1984. 160 p.

REIS, G.N.; FURLANI, C.E.A.; SILVA, R.P.; GERLACH, J.R.; CORTEZ, J.W.; GROTTA, D.C.C. Decomposição de culturas de cobertura no sistema plantio direto, manejadas mecânica e quimicamente. Engenharia Agrícola, Jaboticabal, v.27, n.1, p.194-200, 2007.

RIBEIRO JUNIOR, P. P.; DIGGLE, P. P. GeoR: a package for geoestatistical analysis. $R-$ News, Rochester, v. 1, n. 2, p. 15-18, 2001.

SOUZA, C.F.; FOLEGATTI, M.V.; MATSURA, E.E.; OR, D. Calibração da reflectometria no domínio do tempo (TDR) para a estimativa da concentração da solução no solo. Engenharia Agrícola, Jaboticabal, v.26, n.1, p.282-291, 2006.

SOUZA, Z.M.; CAMPOS, M.C.C.; CAVALCANTE, I.H.L.; MARQUES JÚNIOR, J.; CESARIN, L.G.; SOUZA, S.R. Dependência espacial da resistência do solo à penetração e do teor de água do solo sob cultivo contínuo de cana-de-açúcar. Ciência Rural, Santa Maria, v.36, n.1, p.128-134, 2005.

STONE, L.F.; SILVEIRA, P.M.; MOREIRA, J.A.A. Atributos físico-hídricos do solo sob plantio direto. Santo Antônio de Goiás: Embrapa Arroz e Feijão, 2006. 39 p. (Documentos, Embrapa Arroz e Feijão)

TEDESCO, M.J.; GIANELLO, C.; BISSANI, C. A.; BOHNEN, H.; VOKWEISS, S. J. Análise de solo, plantas e outros materiais. 2.ed. Porto Alegre: UFRGS, 1995. 174 p. (Boletim Técnico, 5).

TEIXEIRA, W. G.; SCHROTH, G.; MARQUES, J. D.; HUWE, B. Sampling and TDR probe insertion in the determination of the volumetric soil water content. Revista Brasileira de Ciência do Solo, Viçosa - MG, v.27, p.575-582, 2003.

VERONESE JÚNIOR, V.; CARVALHO, M. P.; DAFONTE, J.; FREDDI, O. S.; VIDAL VÁZQUEZ, E.; INGARAMO, O. E. Spatial variability of soil water content and mechanical resistance of Brazilian ferralsol. Soil \& Tillage Research, Amsterdan, v.85, n.1, p.166-177, 2006.

WEBSTER, R.; OLIVER, M. Geostatistics for environmental scientists. Chichester: John Wiley \& Sons, 2001. $271 \mathrm{p}$.

WEIRICH NETO, P.H.; BORGHI, E.; SVERZUT, C.B.; MANTOVANI, E.C.; GOMIDE, R.L.; NEWES, W.L.C. Análise multivariada da resistência do solo à penetração sob plantio direto. Ciência Rural, Santa Maria, v.36, n.4, p.1.186-1.192, 2006. 